\title{
Anti-mesothelin CIR mRNA-electroporated Autologous T Cells
}

National Cancer Institute

\section{Source}

National Cancer Institute. Anti-mesothelin CIR MRNA-electroporated Autologous T Cells. NCI Thesaurus. Code C97038.

Autologous chimeric immune receptor (CIR) T cells transfected with anti-mesothelin chimeric T cell receptor mRNA, with potential antineoplastic activity. The anti-mesothelin mRNA encodes a sing le chain antibody variable fragment (ScFv), the intracellular CD 3 zeta T cell receptor domain and the 4-1BB (cd137) costimulatory domain. Upon intravenous administration, the anti-mesothelin CIR mRNA-electroporated autologous T cells may attach to cancer cells expressing mesothelin. This may stimulate the secretion of multiple cytokines and may result in cell lysis of mesothelin-expressing cancer cells. Mesothelin is a cell surface glycoprotein involved in cell adhesion and is overexpressed in many epithelial-derived cancers. 